\title{
AFM, XRD and HRTEM Studies of Annealed FePd Thin Films
}

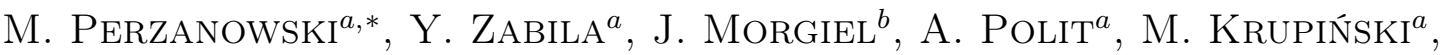 \\ A. Dobrowolska ${ }^{a}$ AND M. MARSZAŁEK ${ }^{a}$ \\ ${ }^{a}$ The H. Niewodniczański Institute of Nuclear Physics, Polish Academy of Sciences \\ Radzikowskiego 152, 31-342 Kraków, Poland \\ ${ }^{b}$ Institute of Metallurgy and Materials Science, Polish Academy of Sciences \\ Reymonta 25, 30-059 Kraków, Poland
}

\begin{abstract}
Ferromagnetic FePd $L 1_{0}$ ordered alloys are highly expected as forthcoming high-density recording materials, because they reveal a large perpendicular magnetocrystalline anisotropy [1]. The value of the magnetic anisotropy of FePd alloy strongly depends on the alloy composition, degree of alloy order as well as on the crystallographic grain orientation. In particular, to obtain the perpendicular anisotropy, it is necessary to get the films with (001) texture. One of the successful methods, which allows one to obtain highly ordered alloy, is a subsequent deposition of Fe and Pd layers, followed by an annealing at high temperature. This paper presents the study of the FePd thin alloy film structure changing in the result of high temperature annealing. During the annealing in high vacuum, the measurements of electrical resistance were performed, indicating the regions of different structure evolution. Changes in the crystal structure and surface morphology induced by thermal treatment were investigated by $\mathrm{X}$-ray diffraction, atomic force microscopy, as well as high resolution transmission electron microscopy and then compared with electrical resistivity measurement. The slow thermal annealing of the deposited layers leads to the formation of $L 1_{0}$ ordered FePd alloy with preferred (111) grain orientation. After the annealing at the highest used temperature, the dewetting process was observed, resulting in a creation of well oriented, regular nanoparticles.
\end{abstract}

PACS numbers: 68.35.bd, 68.55.-a, 73.50.-h, 68.37.Yz

\section{Introduction}

The ordered FePd thin films due to their hard magnetic properties draw high interest in recent times $[1,2]$. Their very large uniaxial magnetocrystalline anisotropy $\left(1.8 \mathrm{MJ} / \mathrm{m}^{3}\right)$ is related to the tetragonal $L 1_{0}$ structure, which occurs in FePd alloy after annealing at high temperature. The FePd $L 1_{0}$ ordered alloy has a lower value of the anisotropy constant than $L 1_{0}$ ordered FePt alloy, but on the other hand, it has also lower annealing temperature at which the $L 1_{0}$ structure appears [3]. These properties make FePd thin alloy films a possible candidate for a new generation of high density magnetic storage devices [4]. The detailed studies of deposition and thermal treatment of $L 1_{0}$ ordered FePd thin alloy films could accelerate the development of the magnetic storage devices with perpendicular magnetic recording. Results of the investigations on thermally treated epitaxial FePd thin films and foils were previously reported in [5]. In this paper we present the influence of slow thermal annealing process on structure and surface morphology of FePd polycrystalline thin films.

* corresponding author; e-mail: Marcin.Perzanowski@ifj.edu.pl

\section{Experimental details}

The $\mathrm{Fe} / \mathrm{Pd}$ multilayers were prepared by alternating thermal evaporation of high-purity metals on (100) oriented Si substrates covered by $100 \mathrm{~nm}$ of amorphous $\mathrm{SiO}_{x}$. Prior to deposition the $\mathrm{SiO}_{x} / \mathrm{Si}(100)$ wafers were ultrasonically cleaned in organic solvents and rinsed in deionized water. The pressure was kept below $5 \times 10^{-8}$ mbar during the evaporation. The deposition rates and layer thicknesses were controlled by calibrated quartz microbalances located close to the substrate position. The deposition rates were in the range $0.005-0.03 \mathrm{~nm} / \mathrm{s}$ for Fe and $\mathrm{Pd}$, respectively. The thicknesses of $\mathrm{Fe}(0.9 \mathrm{~nm})$ and $\mathrm{Pd}(1.1 \mathrm{~nm})$ correspond to the stoichiometric ratio about $1: 1$ which allows one to obtain FePd alloy after annealing. The bilayer $\mathrm{Fe} / \mathrm{Pd}$ was repeated 5 times and the total thickness was equal to $10 \mathrm{~nm}$. After deposition the samples were annealed in high vacuum from room temperature to $550{ }^{\circ} \mathrm{C}$ with annealing rate of $3^{\circ} \mathrm{C} / \mathrm{min}$ and the simultaneous measurement of electrical resistance by two-point contact method was carried out. Structure and surface morphology of the samples were investigated using XRD and AFM methods directly after deposition, as well as after annealing at three different temperatures, chosen in a way de- 
scribed in the next section. The X-ray diffraction measurements were performed with a Philips X'Pert MRD Pro diffractometer. The $\mathrm{Cu}$ radiation operated at $40 \mathrm{kV}$ and $30 \mathrm{~mA}$ was used together with the $K_{\alpha}$ monochromator at the scattered beam. Atomic force microscope images were taken with the Park Xe-120 instrument in noncontact mode at room temperature. Additionally, the structure of the sample annealed at the temperature of $550^{\circ} \mathrm{C}$ was studied by Transmission Electron Microscope Tecnai G2 F20.

\section{Results}

The dependence of resistance on temperature $R(T)$ is shown in Fig. 1. Initially slow linear increase of resistance with temperature was observed up to about $250^{\circ} \mathrm{C}$, called for convenience temperature $T_{1}$. After reaching temperature $T_{1}$ the electrical resistance gradually decreased with the temperature down to the minimum at about $400{ }^{\circ} \mathrm{C}\left(T_{2}\right)$. Next, the drastic increase of the resistance was observed up to the temperature about $500^{\circ} \mathrm{C}\left(T_{3}\right)$, and then the almost constant value of resistance was

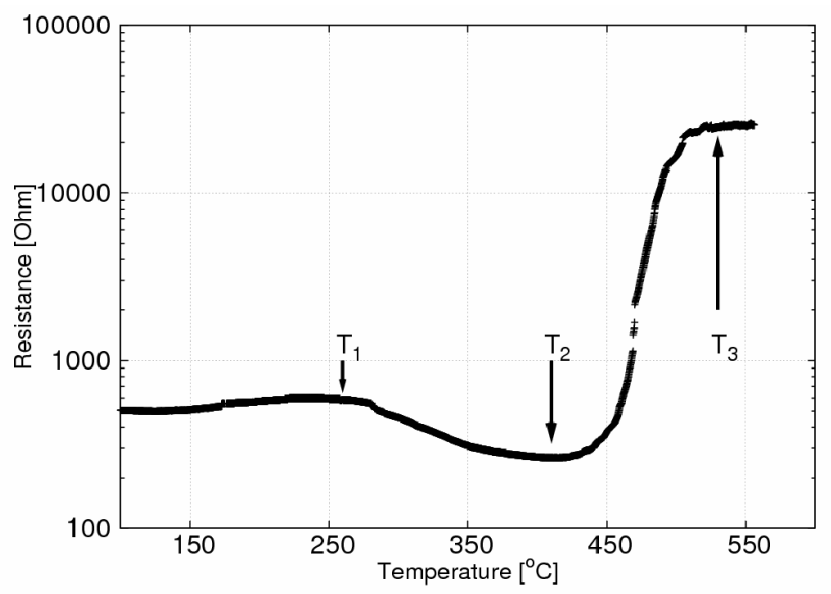

Fig. 1. The electrical resistance dependence on temperature for the annealed FePd thin film. Specific values of the temperature are indicated.

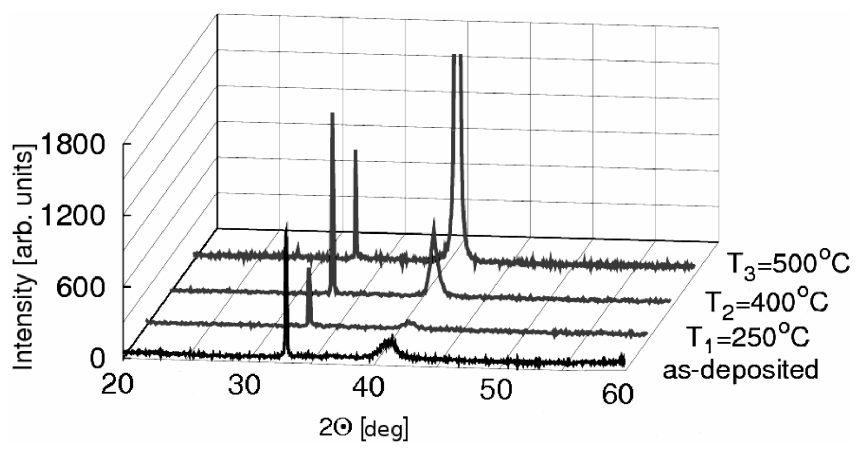

Fig. 2. XRD patterns for as-deposited and annealed at different temperatures FePd thin films. measured. Basing on these results, we annealed in the same conditions two samples, first of them up to temperature $T_{1}$, the next one up to temperature $T_{2}$. After reaching temperature $T_{i}$ each sample was kept at this temperature for $1 \mathrm{~h}$. Figure 2 presents XRD spectra for the sample as-deposited and annealed at temperatures $T_{1}, T_{2}$ and $T_{3}$. In the spectra the peak coming from the Si substrate is seen at $2 \theta=33^{\circ}$. The other peak observed in the diffraction spectra has the position of $2 \theta=41.2^{\circ}$ for as-deposited sample and moves towards $2 \theta=41^{\circ}$ for the annealed samples. It changes intensity from very low value for the sample annealed at $250^{\circ} \mathrm{C}$ to very large value for the sample annealed at $500^{\circ} \mathrm{C}$. Full width at half maximum (FWHM), which is a measure of crystallite size, changes from $1.2^{\circ}$ for the samples as-deposited and annealed at $250{ }^{\circ} \mathrm{C}$, through $0.6^{\circ}$ for the sample annealed at $400^{\circ} \mathrm{C}$, to $0.2^{\circ}$ for the sample annealed at $500^{\circ} \mathrm{C}$. For the as-deposited sample it comes from (111) plane of disordered FePd fcc phase, which occurred on the interfaces between $\mathrm{Fe}$ and $\mathrm{Pd}$ layers because of intermixing.

X-ray reflectivity (XRR) spectrum of as-deposited sample was also taken and it is shown in Fig. 3. The spectrum for $[\mathrm{Fe} / \mathrm{Pd}]_{5}$ multilayer demonstrates well-defined Kiessig fringes, however, the Bragg peak indicating the bilayer thickness and periodicity is not observed, suggesting the significant roughness of $\mathrm{Fe} / \mathrm{Pd}$ interfaces. Indeed, from the detailed analysis of experimental data, done with simulation program REFLECTIVITY of Panalytical using Parrat formalism [6] the obtained roughness of Fe and Pd layers was $0.27 \mathrm{~nm}$ and $0.42 \mathrm{~nm}$, respectively.

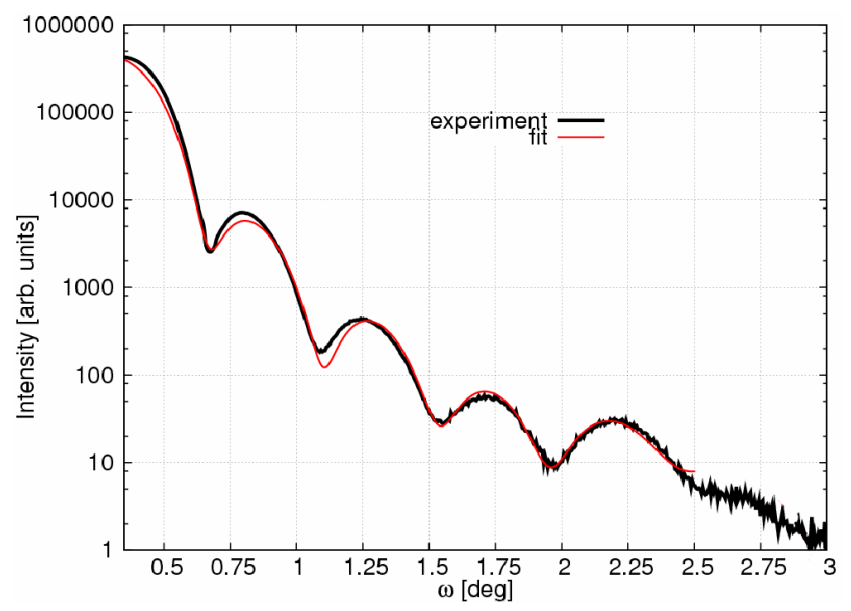

Fig. 3. XRR scan for as-deposited FePd thin film. The experimental data were fitted in the shorter range than experimental data due to the limitations of software.

A good agreement was found between nominal and estimated $\mathrm{Fe} / \mathrm{Pd}$ multilayer total thickness. The density of Fe and Pd films differed not more than $10 \%$ from bulk density values. Figure 4 shows $1.4 \mu \mathrm{m} \times 1.4 \mu \mathrm{m}$ AFM images of as-deposited sample (a) and sample annealed for $1 \mathrm{~h}$ at temperature $500{ }^{\circ} \mathrm{C}$ (b). The calculated roughness of the surface of as-deposited sample equals $0.7 \mathrm{~nm}$. An- 
nealing at temperatures $250^{\circ} \mathrm{C}$ and $400{ }^{\circ} \mathrm{C}$ did not cause any substantial changes of roughness (data not shown here). Surprisingly, the surface of the sample annealed at temperature $500{ }^{\circ} \mathrm{C}$ exhibited the formation of separate islands with the average diameter $200 \mathrm{~nm}$, although smaller (90 nm) and bigger (up to $450 \mathrm{~nm}$ diameter) islands were also observed.

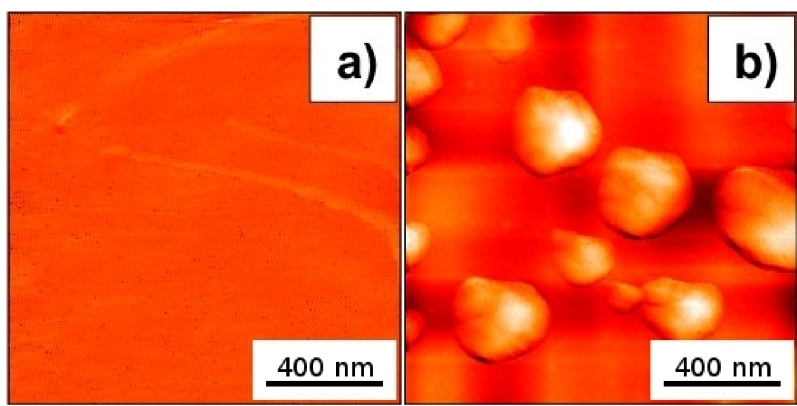

Fig. 4. AFM scans of $1.4 \mu \mathrm{m} \times 1.4 \mu \mathrm{m}$ size: (a) as-deposited sample, (b) sample annealed for $1 \mathrm{~h}$ at $500{ }^{\circ} \mathrm{C}$ temperature.

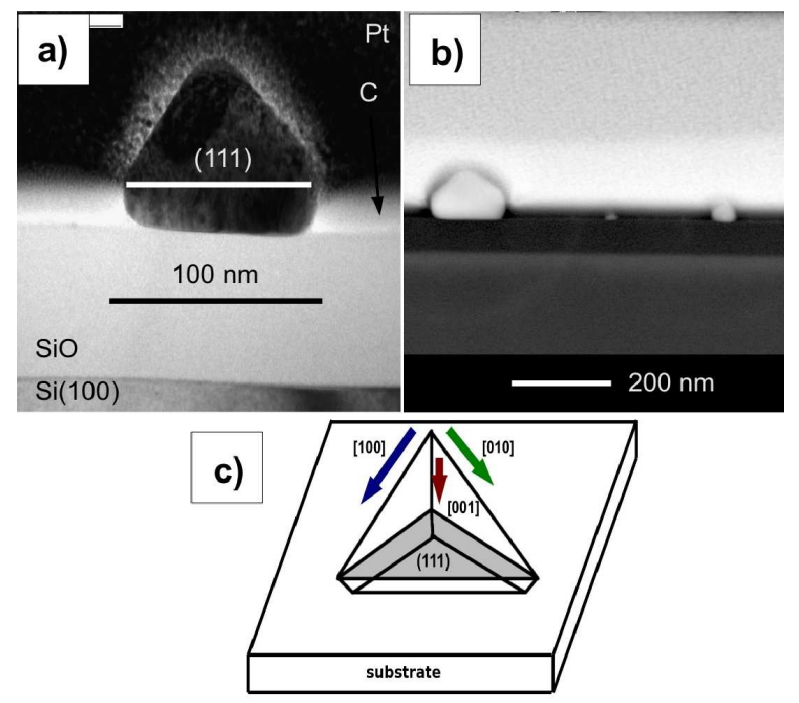

Fig. 5. HRTEM images of the FePd thin film annealed for $1 \mathrm{~h}$ at $500{ }^{\circ} \mathrm{C}$ : (a) transmission mode image of single island, (b) diffraction mode image of island distribution, (c) schematic image showing island shape and orientation. The platinum and carbon layers were deposited before HRTEM measurements for proper image contrast.

Figure 5 shows a cross-section transmission image of the FePd sample annealed at temperature $500{ }^{\circ} \mathrm{C}$ obtained using bright field (5a) and scanning transmission high angle annular dark field (5b) modes. The surface of the sample was covered with islands of diameter about $100 \mathrm{~nm}$ and separated one from the other by $250 \mathrm{~nm}$ with specific tetrahedra-like shape with under-cut at the bottom, schematically presented in Fig. 5c. The space between the islands is bare $\mathrm{SiO}_{x}$ substrate. The structure analysis showed that $\mathrm{FePd}$ islands are homogeneous FePd alloy; no other phases were observed.

\section{Discussion}

The unusual in metallic systems temperature dependence of resistance was observed during annealing of $\mathrm{FePd}$ thin alloy films. It indicates the transformation of the structure which takes place during heating. As already described three regions of resistance changes are observed. After initial small increase of resistance (temperature range $\mathrm{RT}-250^{\circ} \mathrm{C}$ ) the atomic diffusion starts inducing the annealing of defects and ordering of crystallites. The drastic increase of the resistance at temperatures above $400{ }^{\circ} \mathrm{C}$ can suggest instability of structure related to great mobility of atoms. Finally, the plateau reached at $500{ }^{\circ} \mathrm{C}$ and a high value of the resistance is an indication of semiconducting character of sample suggesting the appearance of dewetting process. Similar results were observed in [5] for FePd thin films and foils.

Besides the peak from the substrate the diffraction spectrum of as-deposited sample showed only a weak and broad peak at $2 \theta$ about $41^{\circ}$. This peak corresponds to (111) plane of FePd disordered fcc alloy which occurred on the interfaces between $\mathrm{Fe}$ and $\mathrm{Pd}$ layers because of the intermixing due to significant interface roughness. As the annealing temperature increases, the (111) peak intensity is enhanced but the peak width decreases, which indicates an enhancement in the (111) preferred orientation and the increase in the grain size of $\mathrm{FePd}$ film. Signatures of $L 1_{0}$ phase (fundamental (002) peak and superstructure (001) peak) were not detected, therefore we did not observe the chemically ordered $L 1_{0}$ phase in our samples.

The AFM images clearly show the 2D-3D surface transformation as the annealing temperature increases. The as-deposited sample is very flat with a root mean square roughness of $0.7 \mathrm{~nm}$, whereas the samples annealed at $500{ }^{\circ} \mathrm{C}$ clearly exhibit a nanostructured surface morphology with presence of nanoparticles with an average diameter of $200 \mathrm{~nm}$ and a mean height of $150 \mathrm{~nm}$, with an average aspect ratio of roughly 1 to 1 . The density of islands equals to $600 / 100 \mu \mathrm{m}^{2}$. Since the AFM is not chemically sensitive, the HRTEM images were taken in order to see what is the composition of nanoislands and what kind of material between them is left. These studies confirmed that islands are isolated objects on the $\mathrm{SiO}_{x}$ surface. The conclusion of this experiment is that annealing for $1 \mathrm{~h}$ at $500{ }^{\circ} \mathrm{C}$ leads to the dewetting process which results in formation of regularly shaped nanoislands of FePd alloy, confirming the percolating character of temperature dependence of resistance in this temperature range.

\section{Conclusions}

In the present paper the evolution of FePd thin alloy film microstructure annealed at different temperatures 
is shown. The thermal treatment of FePd alloys has shown the, unusual in metallic systems, temperature dependence of resistance. Between room temperature and $200{ }^{\circ} \mathrm{C}$ a linear dependence of $R(T)$, standard for metals, is seen. Further, the decrease of resistance with temperature up to $400{ }^{\circ} \mathrm{C}$ related to the ordering of removal of defects is observed. The island structure on sample surface, appearing above annealing temperature $500{ }^{\circ} \mathrm{C}$, is responsible for giant changes in electrical resistance. Heating of $\mathrm{Fe} / \mathrm{Pd}$ multilayers results in ordering of crystallite structure, however chemical order is not observed. The dewetting of FePd thin alloy films on $\mathrm{SiO}_{x} / \mathrm{Si}(100)$ substrate is demonstrated, combining the microstructure observations with the temperature changes of electrical resistance. It was observed that a $\mathrm{FePd}$ thin film dewets by spontaneously retracting, forming faceted objects with (111) crystallographic plane parallel to the sample substrate.

\section{Acknowledgments}

This paper was partially supported by the grant $\mathrm{N}$ N507 421136 from Polish Ministry of Science and Higher Education. The support of DAAD-Polish Ministry of Science and Higher Education program of Polish-German cooperation under the contract $329 / \mathrm{N}-\mathrm{DAAD} / 2008 / 0$ is also acknowledged.

\section{References}

[1] S.N. Piramanayagam, J. Appl. Phys. 102, 01130 (2007).

[2] S.N. Piramanayagam, K. Srinivasan, J. Magn. Magn. Mater 321, 485 (2009).

[3] F.M. Takata, G. Pattanaik, W.A. Soffa, P. Sumodjo, G. Zangari, Electrochem. Commun. 10, 568 (2008).

[4] Y. Hirotsu, K. Sato, J. Ceram. Processing Res. 3, 236 (2005).

[5] Ch. Issoro, W. Puschl, W. Pfeiler, P.F. Rogl, W.A. Soffa, M. Acosta, G. Schmerber, R. Kozubski, V. Pierron-Bohnes, Scr. Mater. 53, 447 (2005).

[6] L.G. Parratt, Phys. Rev. 95, 359 (1954). 